\title{
Serum neurofilament light chain in behavioral variant frontotemporal dementia
}

Petra Steinacker, PhD, Sarah Anderl-Straub, PhD, Janine Diehl-Schmid, MD, Elisa Semler, Dipl-Psych, Ingo Uttner, PhD, Christine A.F. von Arnim, MD, Henryk Barthel, MD, Adrian Danek, MD, Klaus Fassbender, MD, Klaus Fliessbach, MD, Hans Foerstl, MD, Timo Grimmer, MD, Hans-Jürgen Huppertz, MD, Holger Jahn, MD, Jan Kassubek, MD, Johannes Kornhuber, MD, Bernhard Landwehrmeyer, MD, Martin Lauer, MD, Juan Manuel Maler, MD, Benjamin Mayer, PhD, Patrick Oeckl, PhD, Johannes Prudlo, MD, Anja Schneider, MD, Alexander E. Volk, MD, Jens Wiltfang, MD, Matthias L. Schroeter, MD, PhD, Albert C. Ludolph, MD, and Markus Otto, MD, on behalf of the FTLDc study group

Neurology ${ }^{\circledR}$ 2018;91:e1390-e1401. doi:10.1212/WNL.0000000000006318

\section{Abstract}

\section{Objective}

To determine the association of serum neurofilament light chain (NfL) with functional deterioration and brain atrophy during follow-up of patients with behavioral variant frontotemporal dementia (bvFTD).

\section{Methods}

Blood NfL levels from 74 patients with bvFTD, 26 with Alzheimer disease (AD), 17 with mild cognitive impairment (MCI), and 15 healthy controls (Con) at baseline and follow-up were determined and analyzed for the diagnostic potential in relation to functional assessment (Clinical Dementia Rating Scale Sum of Boxes [CDR-SOB], frontotemporal lobar degeneration-related CDR-SOB, Mini-Mental State Examination $[\mathrm{MMSE}])$ and brain volumetry.

\section{Results}

At baseline, serum NfL level correlated with CSF NfL (bvFTD $r=0.706, p<0.0001 ; \mathrm{AD} / \mathrm{MCI} r=0.666, p=$ 0.0003 ). Highest serum levels were observed in bvFTD ( $p<00.0001$ vs Con and MCI, $p=0.0078$ vs AD, respectively). Discrimination of bvFTD from Con/MCI/AD was possible with $91 \% / 74 \% / 74 \%$ sensitivity and 79\%/74\%/58\% specificity. At follow-up, serum NfL increased in bvFTD and $\mathrm{AD}$ ( $p=0.0039$ and $p=$ 0.0006 , respectively). At baseline and follow-up, NfL correlated with functional scores of patients with bvFTD (e.g., CDR-SOB [baseline] $r=0.4157, p=0.0006$; [follow-up] $r=0.5629, p<0.0001$ ) and with atrophy in the gray and white matter of many brain regions including frontal and subcortical areas (e.g., frontal lobe: $r=$ $-0.5857, p<0.0001$; $95 \%$ confidence interval -0.7415 to -0.3701 ). For patients with $\mathrm{AD} / \mathrm{MCI}$, NfL correlated with the functional performance as well (e.g., CDR-SOB [baseline] $r=0.6624, p<0.0001$; [followup] $r=0.5659, p=0.0003$ ) but not with regional brain volumes.

\section{Conclusions}

As serum NfL correlates with functional impairment and brain atrophy in bvFTD at different disease stages, we propose it as marker of disease severity, paving the way for its future use as outcome measure for clinical trials.

\section{Classification of evidence}

This study provides Class III evidence that for patients with cognitive problems, serum NfL concentration discriminates bvFTD from other forms of dementia.

\author{
Correspondence \\ Dr. Otto \\ markus.otto@uni-ulm.de
}

\section{MORE ONLINE}

$\rightarrow$ Class of Evidence

Criteria for rating

therapeutic and diagnostic studies

NPub.org/coe 


\section{Glossary}

$\mathbf{A} \beta 42=\beta$-amyloid 1-42; AD = Alzheimer disease $; \mathrm{ALS}=$ amyotrophic lateral sclerosis; $\mathbf{A U C}=$ area under the curve; $\mathbf{b v F T D}=$ behavioral variant frontotemporal dementia; CDR-SOB = Clinical Dementia Rating scale Sum of Boxes; CI $=$ confidence interval; FTD = frontotemporal dementia; $\mathbf{F T L D}=$ frontotemporal lobar degeneration; $\mathbf{I C V}=$ intracranial volume; $\mathbf{M C I}=$ mild cognitive impairment; $\mathbf{M M S E}=$ Mini-Mental State Examination; NfL = neurofilament light chain; $\mathbf{p}$-tau = phosphorylated tau; $\mathbf{p N f H}=$ phosphorylated neurofilament heavy chain; $\mathbf{R O C}=$ receiver operating characteristic; $\mathbf{v} \mathbf{1}=$ visit $\mathbf{1} ; \mathbf{v} \mathbf{2}=$ visit 2.

Frontotemporal dementia (FTD), a form of dementia affecting people under the age of 65 years, ${ }^{1}$ represents a clinically and neuropathologically heterogeneous group of diseases within the spectrum of frontotemporal lobar degenerations (FTLD). The most frequent form is behavioral variant FTD (bvFTD), which is characterized by progressive changes in personality and behavior, often expressed as apathy and disinhibition. Cognitive deficits can be seen as deficits of executive functions while memory is largely preserved. Neuroimaging of frontal brain region atrophy, starting in the frontoinsular cortices and further regions of the medial paralimbic network, is included in the revised diagnostic criteria for probable bvFTD ${ }^{2,3}$ and has been demonstrated in comprehensive imaging meta-analyses. ${ }^{4,5}$ At the neuropathologic level, $90 \%$ of bvFTD cases are characterized by tau or TDP- 43 pathology in equal shares, and about $10 \%$ show FUS pathology. ${ }^{6,7}$

At present, in bvFTD only behavioral symptoms are treated pharmacologically, e.g., some patients benefit from selective serotonin reuptake inhibitors, ${ }^{8}$ but there is no medication available targeting the underlying pathogenesis. Our knowledge about pathomechanisms, however, has substantially increased within the last decade, giving hope that neuroprotective etiology-specific treatment will be available in the foreseeable future. When available, it is important to have tools, such as neurochemical biomarkers, to monitor disease progression and therapy effects in patients.

Neurofilament light chain (NfL) is a component of the neuronal cytoskeleton released into CSF and blood upon neurodegeneration. Increased CSF levels have been reported for amyotrophic lateral sclerosis (ALS) and some of the FTLD spectrum diseases, which pathophysiologically are closely related, but also for other diseases, e.g., Alzheimer disease $(\mathrm{AD})$, multiple sclerosis, or traumatic brain injury. ${ }^{9-13}$

The recent development of a sensitive electrochemiluminescence immunoassay and in particular the establishment of an ultrasensitive single-molecule immunoassay for the measurement of NfL now enables us to reliably quantify NfL in serum, ${ }^{14,15}$ resulting in the first reports on increased levels in neurodegenerative diseases, including those of the ALS-FTLD spectrum, in part correlating with disease severity and progression. ${ }^{12,16-24}$

In the present study, we aimed to determine the potential of blood NfL levels as a measure to objectively monitor disease progression in bvFTD. We considered subgroups clinically diagnosed with possible bvFTD and probable bvFTD since clinical trials most likely will initially include the latter group, and compared our results with those from patients with $\mathrm{AD}$ and patients with mild cognitive impairment (MCI), as well as healthy controls, as reference groups to examine the specificity of blood NfL.

\section{Methods}

The primary research question was the following: Do serum NfL levels discriminate bvFTD from $\mathrm{AD}, \mathrm{MCI}$, and controls (Class III level of evidence)?

\section{Standard protocol approvals, registrations, and patient consents}

The study was conducted according to the principles expressed in the Declaration of Helsinki. Collection and analysis of samples were approved by the local ethics committees of Departments of Neurology and Psychiatry participating in the German FTLD Consortium, a qualitycontrolled, monitored, multicenter initiative (Ulm approval number 20/10). ${ }^{25}$ All patients or their legal relatives in case of severe dementia gave written informed consent to their participation in the study.

\section{Participants}

A total of 132 patients were included in this prospective study. Patients for whom at least one in-depth follow-up examination with serum sampling after a regular interval of 1 year $( \pm 3$ months) was available were included in our study. Demographic and disease data are given in tables 1 and 2 .

The bvFTD group consisted of 74 patients including 27 possible and 38 probable bvFTD cases diagnosed according to international diagnostic criteria. ${ }^{2,3}$ Genetic screening of 67 patients with bvFTD for C9orf 72 repeat expansion revealed 7 carriers. Analysis of MAPT and GRN in 18 patients revealed 1 carrier of the MAPT:c.1008 G>C (p.Gln336His) mutation and 1 carrier of the GRN: c.349+1G>C mutation. The 9 patients carrying mutations were categorized as definite bvFTD. Disease duration at baseline, defined as interval between the date of first symptoms reported by the patient or caregiver and the date of blood/CSF sampling, ranged from 4 months to 13.4 years. CSF samples were taken at baseline from 37 patients with bvFTD ( 15 possible, 17 probable, and 5 definite) and for none of these was a biomarker pattern typical for $\mathrm{AD}$ ( $\beta$-amyloid 1-42 $[\mathrm{A} \beta 42]<550 \mathrm{pg} / \mathrm{mL}$, tau $>450 \mathrm{pg} /$ 
Table 1 Demographic characteristics, functional performance, brain volumetry, and serum neurofilament light chain (NfL) of the study subgroups at baseline

\begin{tabular}{|c|c|c|c|c|c|}
\hline & bvFTD & $A D$ & $\mathrm{MCl}$ & Controls & $p$ Value \\
\hline No. & 74 & 26 & 17 & 15 & \\
\hline F/M & $30 / 44$ & $11 / 15$ & $4 / 13$ & $9 / 6$ & \\
\hline Age, y, mean (SD) & $63.7(9.2)$ & $67(8.1)$ & $63.1(9.3)$ & $64.8(11.3)$ & 0.3540 \\
\hline Disease duration, $y$, mean (SD) & $3.9(3.4)$ & $3.4(2.1)$ & $3.6(3.2)$ & NA & 0.8356 \\
\hline Serum NfL, pg/mL, mean (SD) & $49.0(35.2)$ & $32.3(15.8)$ & $16.6(8.1)$ & $21.7(20.6)$ & $<0.0001^{a}$ \\
\hline No. & 69 & 26 & 17 & 15 & \\
\hline MMSE, mean (SD) & $24.2(5.2)$ & $21.6(6.0)$ & $27.9(1.9)$ & $29(0.8)$ & $<0.0001^{\text {b }}$ \\
\hline No. & 64 & 22 & 16 & 15 & \\
\hline CDR-SOB, mean (SD) & $6.5(4.6)$ & $5.1(2.4)$ & $0.75(0.7)$ & $0.1(0.2)$ & $<0.0001^{\mathrm{c}}$ \\
\hline FTLD-CDR-SOB, mean (SD) & $8.8(5.7)$ & $6.6(3.3)$ & $0.9(0.9)$ & $0.1(0.2)$ & $<0.0001^{c}$ \\
\hline No. & 54 & 21 & 16 & 12 & \\
\hline Cerebrum, mL, mean (SD) & $818(63.9)$ & $803(44.5)$ & $877(51)$ & $873(69)$ & $0.0001^{d}$ \\
\hline Frontal lobe, $\mathrm{mL}$, mean (SD) & $257(33.3)$ & $265(18.9)$ & $292(19.8)$ & $292(16.4)$ & $<0.0001^{\mathrm{e}}$ \\
\hline Temporal lobe, mL, mean (SD) & $161(20.0)$ & $152(14.0)$ & $176(11,4)$ & $177(9.3)$ & $0.0001^{f}$ \\
\hline No. & 38 & 16 & 9 & & \\
\hline CSF NfL, pg/mL, mean (SD) & $2,948(2,630)$ & $1,595(1,005)$ & $1,110(691)$ & & $0.0036^{\mathrm{g}}$ \\
\hline CSF pNfH, pg/mL, mean (SD) & $446(248)$ & $340(145)$ & $262(128)$ & & $0.0185^{h}$ \\
\hline CSF tau, pg/mL, mean (SD) & $461(413)$ & $659(268)$ & $299(144)$ & & $0.0009^{i}$ \\
\hline CSF p-tau, pg/mL, mean (SD) & $60.6(35.8)$ & $78.1(34.0)$ & $50.0(25,9)$ & & $0.0365^{j}$ \\
\hline CSF A $342, \mathrm{pg} / \mathrm{mL}$, mean (SD) & $941(375)$ & $545(226)$ & $781(291)$ & & $0.0007^{k}$ \\
\hline Aß42-positive samples, ${ }^{\prime} \%$ & 21.0 & 68.8 & 33.3 & & \\
\hline
\end{tabular}

Abbreviations: $A \beta 42$ = $\beta$-amyloid 1-42; $A D$ = Alzheimer disease; bvFTD = behavioral variant frontotemporal dementia; CDR-SOB = Clinical Dementia Rating Sum of Boxes; FTLD = frontotemporal lobar degeneration; $\mathrm{MCl}$ = mild cognitive impairment; MMSE = Mini-Mental State Examination; NA = not applicable; $\mathrm{p}-$ tau = phosphorylated tau; pNfH = phosphorylated neurofilament heavy chain.

The Kruskal-Wallis test was applied to all study subgroups. For statistical differences of CSF measures, the control group was omitted because of the low patient number tested.

a bvFTD vs $\mathrm{AD} p<0.01$, vs $\mathrm{MCl} p<0.001$, vs controls $p<0.001 ; \mathrm{AD}$ vs $\mathrm{MCl} p<0.05$.

${ }^{b}$ bvFTD vs $A D p<0.05$, vs controls $p<0.01$; AD vs $\mathrm{MCI} p<0.001, \mathrm{AD}$ vs controls $p<0.001$.

${ }^{c}$ bvFTD vs $\mathrm{MCl} p<0.001$, vs controls $p<0.0001$; AD vs $\mathrm{MCl} p<0.001$, vs controls $p<0.001$

${ }^{d}$ bvFTD vs $\mathrm{MCl} p<0.05$, vs controls $p<0.01$; AD vs $\mathrm{MCl} p<0.01$, vs controls $p<0.001$.

e bvFTD vs $\mathrm{MCl} p<0.05$, vs controls $p<0.001$; AD vs controls $p<0.05$.

${ }^{\mathrm{f}} \mathrm{AD}$ vs $\mathrm{MCl} p<0.001$, vs controls $p<0.01$.

${ }^{\prime}$ bvFTD vs $\mathrm{MCl} p<0.01$

h bvFTD vs $\mathrm{MCl} p<0.05$.

' bvFTD vs AD $p<0.01$; AD vs $\mathrm{MCl} p<0.01$.

j Post hoc test not significant.

${ }^{k}$ bvFTD vs AD $p<0.001$

${ }^{\mathrm{A}} \mathrm{A} \beta 42<550 \mathrm{pg} / \mathrm{mL}$.

$\mathrm{mL}$, phosphorylated tau $[\mathrm{p}$-tau $]>61 \mathrm{pg} / \mathrm{mL}$ ) determined. For 24 patients with bvFTD ( 2 definite, 10 probable, and 12 possible cases), at least a second follow-up examination (visit 3) was available.

The reference cohort consisted of 26 patients with $\mathrm{AD}^{26} 17$ patients with $\mathrm{MCI},{ }^{27}$ and 15 controls without neurologic disease and without acute or chronic inflammation of the brain. Genetic assessment for $C 9$ orf 72 of 19 patients with $\mathrm{AD}, 11$ patients with
$\mathrm{MCI}$, and 8 controls, and for MAPT and GRN of 7 patients with $\mathrm{AD}, 1$ patient with $\mathrm{MCI}$, and 2 controls, revealed no carrier of repeat expansion or mutation, respectively. CSF samples from 16 patients with $\mathrm{AD}$ and 9 patients with $\mathrm{MCI}$ could be taken at first examination. Out of the $16 \mathrm{AD}$ samples, 8 had a typical CSF $\mathrm{AD}$ core marker pattern. A total of 26 patients with $\mathrm{AD}, 17$ patients with $\mathrm{MCI}$, and 14 controls had at least a second follow-up examination. No patient of the $\mathrm{MCI}$ group converted to $\mathrm{AD}$ or any other dementia within the study interval. 
Table 2 Serum neurofilament light chain (NfL) at follow-up and measures of disease progression in the study subgroups

\begin{tabular}{|c|c|c|c|c|c|}
\hline & bvFTD & AD & $\mathrm{MCl}$ & Controls & $p$ Value \\
\hline Time between v1 and v2, mo, mean (SD) & $12.9(2.3)$ & $13.1(2.2)$ & $12.0(2.5)$ & $13.1(2.7)$ & 0.5535 \\
\hline Serum $\mathrm{NfL}$ at $\mathrm{v} 2, \mathrm{pg} / \mathrm{mL}$, mean (SD) & $56.9(40.3)$ & $38.1(20.6)$ & $21.5(18.9)$ & $17(7.3)$ & $<0.0001^{\circ}$ \\
\hline Change in serum $\mathrm{NfL}, \mathrm{pg} / \mathrm{mL}$, mean (SD) & $8(26.9)$ & $5.8(7.9)$ & $4.9(15.4)$ & $-3.4(21)$ & 0.2789 \\
\hline No. & 64 & 26 & 17 & 14 & \\
\hline Change in MMSE, mean (SD) & $-4.4(6)$ & $-4.5(4.5)$ & $-0.2(2.2)$ & $-0.4(1.7)$ & $0.0002^{\mathrm{k}}$ \\
\hline No. & 61 & 19 & 15 & 13 & \\
\hline Change in CDR-SOB, mean (SD) & $2.3(3.6)$ & $1.8(2.6)$ & $0.3(1.1)$ & $-0.04(0.2)$ & $0.0003^{c}$ \\
\hline No. & 60 & 19 & 15 & 13 & \\
\hline Change in FTLD-CDR-SOB, mean (SD) & $2.9(4,6)$ & $2.4(3)$ & $0.5(1.5)$ & $-0.04(0.2)$ & $0.0004^{\circ}$ \\
\hline No. & 33 & 13 & 13 & 12 & \\
\hline Cerebrum atrophy, \%, mean (SD) & $-1.8(2.2)$ & $-1.9(3.4)$ & $-0.3(2.9)$ & $-0.07(1.4)$ & $0.0418^{\mathrm{e}}$ \\
\hline Frontal lobe atrophy, \%, mean (SD) & $-3(4.8)$ & $-2.5(4.8)$ & $-0.3(2.8)$ & $0.3(1.8)$ & $0.0316^{\epsilon}$ \\
\hline Temporal lobe atrophy, \%, mean (SD) & $-2.2(3)$ & $-1.3(3.1)$ & $0.04(3.5)$ & $-0.7(2)$ & 0.1633 \\
\hline
\end{tabular}

Abbreviations: $A D=$ Alzheimer disease; bvFTD = behavioral variant frontotemporal dementia; $C D R-S O B=$ Clinical Dementia Rating scale Sum of Boxes; FTLD = frontotemporal lobar degeneration; $\mathrm{MCl}=$ mild cognitive impairment; $\mathrm{MMSE}=$ Mini-Mental-State Examination; $\mathrm{v} 1$ = visit 1; v2 = visit 2 .

${ }^{a}$ bvFTD vs $\mathrm{MCl} p<0.001$, vs controls $p<0.001 ; \mathrm{AD}$ vs $\mathrm{MCl} p<0.05$, vs controls $p<0.01$.

${ }^{b}$ bvFTD vs MCI $p<00.01$, vs controls $p<0.05$; AD vs MCl $p<0.01$, vs controls $p<0.01$.

' bvFTD vs $\mathrm{MCl} p<0.05$, vs controls $p<0.01$; AD vs controls $p<0.05$.

${ }^{d}$ bvFTD vs $\mathrm{MCl} p<0.05$, vs controls $p<0.05 ; \mathrm{AD}$ vs $\mathrm{MCl} p<0.05$, vs controls $p<0.05$

e Post hoc test not significant.

Assessment of all patients at baseline and follow-up investigations included a standardized clinical-neurologic examination, MRI, and functional characterization by means of global rating scales (Mini-Mental State Examination [MMSE], Clinical Dementia Rating scale Sum of Boxes [CDR-SOB], and FTLD-specific CDR-SOB).

\section{MRI and volumetric analyses}

Datasets from baseline and at least one follow-up visit were available for 33 patients with bvFTD (12 possible, 17 probable, and 4 definite bvFTD), 13 patients with $\mathrm{AD}, 13$ patients with MCI, and 12 control participants. 3T MRI data including 3D T1 magnetization-prepared rapid gradient echo sequences were recorded according to standardized protocols. The volumes of different brain regions were determined as described elsewhere. ${ }^{20,28,29}$

\section{Laboratory markers}

Serum was extracted from blood, aliquoted, and stored within 2 hours at $-80^{\circ} \mathrm{C}$ until analysis. CSF was obtained by lumbar puncture and processed likewise. All analyses were performed in a blinded manner. CSF examination included measurement of NfL (IBL, Hamburg, Germany), phosphorylated Nf heavy chain (pNfH) (Biovendor, Heidelberg, Germany), tau and p-tau, and A $\beta 42$ (Fujirebio, Hanover, Germany). Serum NfL was measured by single-molecule assay (Quanterix, Lexington, MA). Intra-assay and interassay variability was determined by measuring the same 3 samples as triplicates on 3 separate plates: intra-assay: $<6.4 \%$ (mean $4.1 \%$ ), interassay: $<15.9 \%$ (mean $10.5 \%$ ). Stability was ensured for up to 4 freeze-thaw cycles and up to 48 hours storage at room temperature (variability $<11 \%$ ).

\section{Statistical analysis}

Nonparametric tests were applied in all analyses. Two groups of measures were analyzed for significant differences by Mann-Whitney test. Three or more groups were analyzed by Kruskal-Wallis test, followed by Dunn post hoc test in case of significant results. Correlation of follow-up serum NfL levels was analyzed by Wilcoxon matched pairs test. $p<0.05$ Was regarded as significant.

The optimal cutoff level for dichotomizing values was selected as the situation maximizing the Youden index. The receiver operating characteristics (ROC) curve is used for a graphic visualization of the effect of the variation in the cutoff values.

All results of atlas-based volumetry were corrected (by division) for individual intracranial volume (ICV) of each participant and then normalized to an ICV of $1,400 \mathrm{~mL}$, which corresponds to the approximate average ICV of healthy adult controls. To compare the progression of brain atrophy, either absolute differences in the ICV-normalized volumes were used or volumes measured at follow-up were normalized to the volumes at baseline and expressed as percentage volume change. 
To determine correlation of measures, Spearman rank coefficient was applied considering $p<0.05$ significant. For the analysis of correlation of serum NfL with 201 regional brain volumes, Bonferroni correction for multiple comparisons was applied and $p<0.0025$ was considered significant.

All analyses were performed using GraphPad (La Jolla, CA) Prism 5.0 software.

\section{Data availability}

Any data not published within the article will be shared in pseudonymized form by request from any qualified investigator for purposes of replicating procedures.

\section{Results}

\section{Description of the cohort at baseline examination}

Demographic characteristics, functional performance, brain volumetric measures, and CSF biomarker concentrations for the study groups bvFTD, $\mathrm{AD}, \mathrm{MCI}$, and controls at baseline are given in table 1 .

Measures did not differ comparing the bvFTD subgroups (possible bvFTD, probable bvFTD, definite bvFTD); therefore all analyses were applied to a single group bvFTD.

The bvFTD, AD, MCI, and controls groups did not differ with respect to age $(p=0.345)$ and the disease duration was comparable in the diseased study groups $(p=0.8356)$. Functional constraints quantified by means of MMSE were more severe in the $\mathrm{AD}$ group ( $p<0.05$ compared to bvFTD, $p$ $<0.001$ compared to MCI and controls, respectively) than in the bvFTD group $(p<0.01$ compared to controls, not significant vs MCI). Assessment by CDR-SOB or FTLD-CDR$S O B$ revealed a more severe impairment for bvFTD or $A D$ vs MCI or controls $(p<0.001)$.

The mean volume of the cerebrum was different in the groups, with lower values in the bvFTD and $\mathrm{AD}$ groups compared to MCI and controls (figure 1A). The differences were more pronounced when looking at regions typically affected in bvFTD and $\mathrm{AD}$ : comparing the diseased groups, the frontal lobe volume was lowest in bvFTD (not significant compared to $\mathrm{AD}, p<0.001$ compared to $\mathrm{MCI}$ ) and the hippocampal volume was lowest in $\mathrm{AD}$ ( $p<0.05$ vs bvFTD and $p<0.001$ vs MCI). Temporal lobe volumes were smallest in $\mathrm{AD}(p<$ 0.001 vs MCI and $p<0.01$ vs controls), but not smaller if compared to bvFTD (table 1). All baseline regional brain volumes for the study groups are given in table e- 1 .

In CSF samples (bvFTD, $\mathrm{AD}$, and $\mathrm{MCI}$ ), neurofilaments ( $\mathrm{NfL}$ and $\mathrm{pNfH}$ ) and the $\mathrm{AD}$ biomarker panel (tau, p-tau, $\mathrm{A} \beta 42$ ) were measured (table 1). Neurofilaments were highest in the bvFTD group ( $p<0.05$ vs $\mathrm{AD}, p<0.01$ vs $\mathrm{MCI}$ ), patients with $\mathrm{AD}$ showed intermediate levels, and patients with MCI had the lowest levels (AD vs MCI not significant). For tau and $\mathrm{p}$-tau, the levels were highest for $\mathrm{AD}$ and similar for bvFTD and controls (tau: $p<0.01$ for bvFTD vs AD and $p$ $<0.001$ for $\mathrm{AD}$ vs MCI; $\mathrm{p}$-tau: $p=0.0365$, not significant in the post hoc test). A marked reduction of $A \beta 42$ was seen for patients with $\mathrm{AD}$ and in patients with MCI A $\beta 42$ also tended to have decreased levels, while the bvFTD group was characterized by a high variability of $A \beta 42$ with a mean value in the range of healthy individuals (bvFTD vs $\mathrm{AD} p<0.001$ ). A considerable number of patients in our $\mathrm{AD}$ group had normal A 342 (31\%), normal tau (25\%), or normal p-tau levels (31\%). Among those with normal $A \beta 42,2$ patients had tau or $p$-tau below the cutoff. In the bvFTD group, $21 \%$ of patients had reduced $A \beta 42$, and 7 of these also had increased p-tau or tau levels.

The serum NfL level was highest for patients with bvFTD, followed by $\mathrm{AD}, \mathrm{MCI}$, and controls (figure 1B).

\section{Baseline serum NfL of patients with bvFTD, diagnostic performance, and correlation with disease measures}

Based on the serum NfL level, patients with bvFTD could be differentiated from controls with 91\% sensitivity (95\% confidence interval [CI] 81\%-96\%) and 79\% specificity (95\% CI $49 \%-95 \%$ ) at a cutoff of $19.5 \mathrm{pg} / \mathrm{mL}$. For the discrimination of bvFTD from $\mathrm{AD}$ and $\mathrm{MCI}$, the highest index was calculated for a cutoff of $29.5 \mathrm{pg} / \mathrm{mL}$, yielding $74 \%$ sensitivity (95\% CI 63\%-84\%) with $58 \%$ and $74 \%$ specificity, respectively (figure $1 \mathrm{C}$ ).

Serum NfL correlated for the bvFTD group with CSF NfL $(r=0.706, p<0.0001)$ and CSF tau $(r=0.4979, p=0.0015)$ (figure 1D) but not with CSF pNfH $(r=0.2689, p=$ $0.1026)$, p-tau $(r=0.1897, p=0.2409)$, or Aß42 $(r=$ $-0.1136, p=0.4852$ ). While there was no correlation of serum NfL with the patient's age or disease duration (table $\mathrm{e}-2)$, it correlated with results of functional assessment (CDR-SOB: $r=0.4157, p=0.0006$, 95\% CI 0.1821 to 0.6050; MMSE: $r=-0.4114, p=0.0004,95 \% \mathrm{CI}-0.5952$ to -0.1866; FTDL-CDR-SOB: $r=0.4175, p=0.0006,95 \% \mathrm{CI}$ 0.1841 to 0.6464 ) (figure $1 \mathrm{D}$ ).

The volumes of a number of brain regions correlated with the level of serum NfL. Results that remained significant after Bonferroni correction for multiple comparisons were obtained for the frontal lobe (figure 1E) and multiple subregions of it, while regional volumes of the temporal lobe showed a weaker association with serum NfL, and parietal or occipital regions did not correlate with NfL. A correlation with serum NfL was observed for striatal and limbic system regions (figure $1, \mathrm{G}$ and $\mathrm{H}$ ) and also for white matter volumes (figure 1I). A summary of correlation analysis results for serum NfL at baseline with demographic, disease, and CSF measures and with regional brain volumes is given in table e-2. 
Figure 1 Serum neurofilament light chain (NfL) levels and correlation with functional scores and brain volumetric results at baseline examination

A. Cerebrum

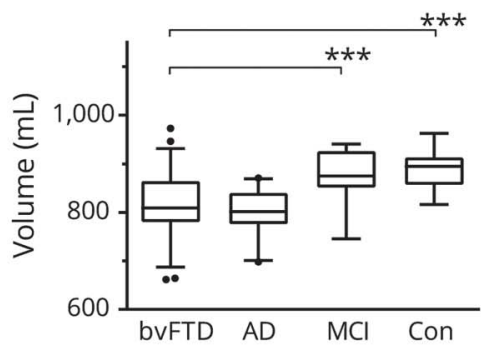

D. CSF-NfL

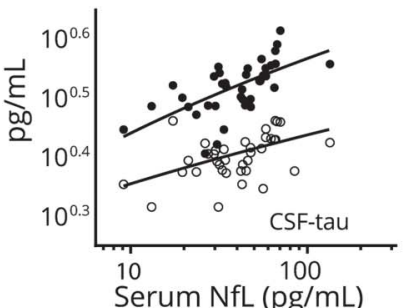

G. Striatum

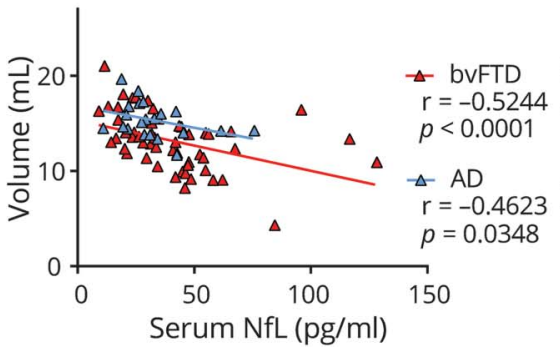

H. Right amygdala

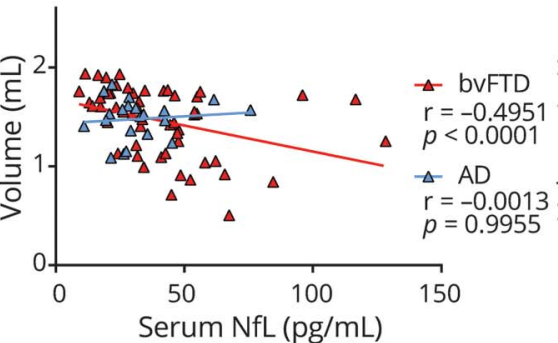

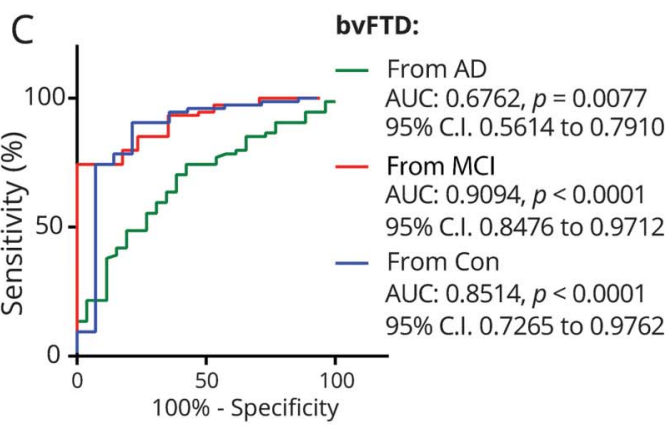

F. Frontal lobe

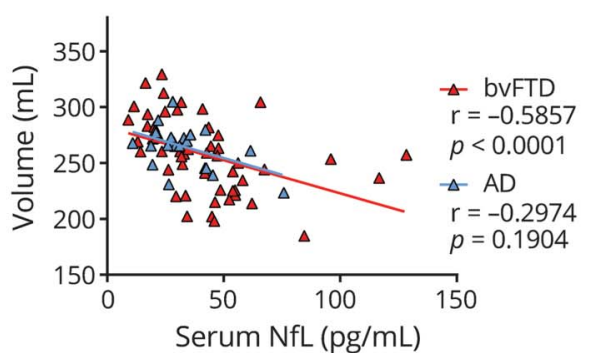

I. Frontal lobe white matter

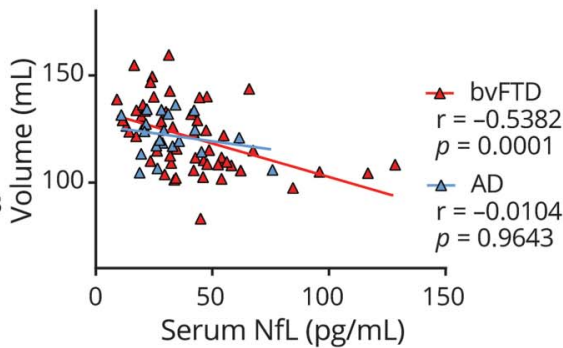

(A) Intracranial volume-normalized cerebrum volumes and (B) serum NfL levels of the diagnostic groups. Boxes show 25 th and 75 th percentile values with median values, whiskers indicate 5th and 95th percentile values, asterisks indicate significant differences determined by post hoc testing after Kruskal-Wallis analysis ( $* p<0.05, * \star p<0.01, * \star \star p<0.001)$. (C) Receiver operating characteristic curves for the discrimination of behavioral variant frontotemporal dementia (bvFTD) from Alzheimer disease (AD), mild cognitive impairment (MCI), or controls. (D) Correlation and linear regression of log-transformed concentrations of serum NfL with CSF NfL (filled circles) and CSF tau (open circles) for bvFTD. (E) Correlation of serum NfL with frontotemporal lobar degeneration Clinical Dementia Rating scale Sum of Boxes (FTLD-CDR-SOB) Score for bvFTD (left y axis, red) and with Mini-Mental State Examination (MMSE) for AD (right y axis, blue). (F-I) Correlation between serum NfL and volumes of the frontal lobe, striatum, right amygdala, and white matter of the frontal lobe. Red indicates bvFTD data, blue indicates $\mathrm{AD}$. $\mathrm{AUC}=$ area under the $\mathrm{curve}$; $\mathrm{Cl}=$ confidence interval.

\section{Baseline serum NfL of patients with AD and patients with $\mathrm{MCl}$ and correlation with disease measures}

In contrast to bvFTD, in the $\mathrm{AD}$ group, serum $\mathrm{NfL}$ correlated with the patient's age at examination and the age at reported symptom onset (age at baseline: $r=0.6857, p=0.0006,95 \%$ CI 0.3488 to 0.8657 ; age at onset: $r=0.7771, p<0.0001,95 \%$ CI 0.4993 to 0.9100$)$. Serum NfL correlated with the CDRSOB ( $r=0.4402, p=0.04,95 \%$ CI 0.0095 to 0.7332$)$ and the FTLD-CDR-SOB score $(r=0.5297, p=0.0112$, 95\% CI 0.1261 to 0.7829$)$, but not with the MMSE score $(r=$ $-0.3242, p=0.161,95 \% \mathrm{CI}-0.694$ to 0.0843$)$ for patients with $\mathrm{AD}$. In the $\mathrm{MCI}$ group, scores did not correlate with serum NfL.
Some regional brain volumes of the $\mathrm{AD}$ and the $\mathrm{MCI}$ group correlated with the serum NfL level, with the association failing to remain after correction for multiple comparisons (table e-2).

Looking at CSF markers, there was no correlation to serum $\mathrm{NfL}$ in the $\mathrm{AD}$ or $\mathrm{MCI}$ group. Defining $\mathrm{AD}$ and $\mathrm{MCI}$ patients together as neurologic control group, serum NfL correlated with CSF NfL $(r=0.6662, p=0.003,95 \%$ CI 0.357 to 0.8438).

\section{Serum NfL level at follow-up examination}

Characteristics of disease progression at follow-up in the bvFTD and the control groups are given in table 2 . 
Figure 2 Serum neurofilament light chain (NfL) at follow-up visits (visit 1 [v1]-visit 3 [v3]) and correlation with functional and morphologic measures
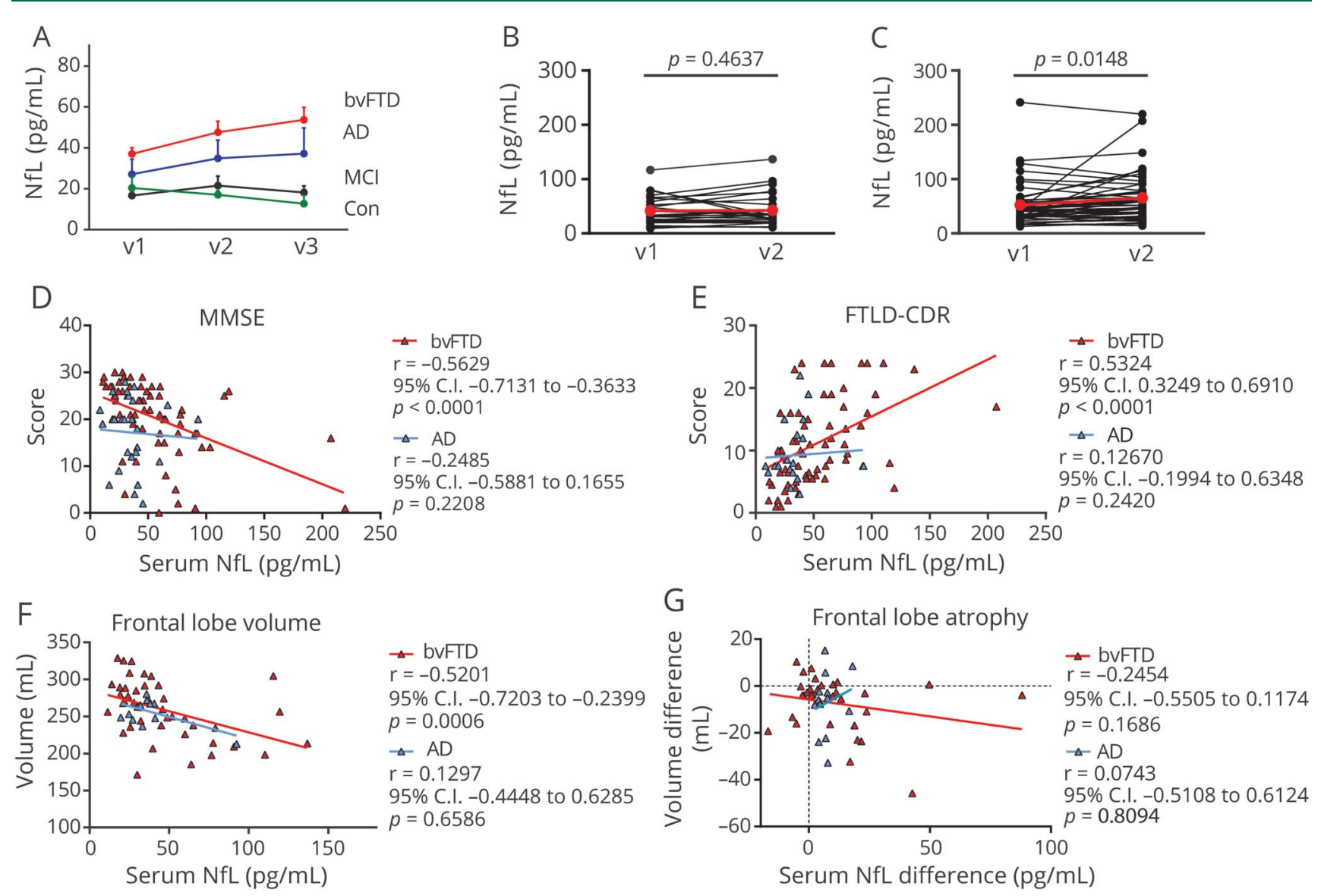

(A) Change of mean serum NfL concentration from baseline examination ( $\mathrm{v} 1$ ) to follow-up 2 years later ( $\mathrm{V} 3$ ). Bars represent the $95 \%$ confidence interval (Cl). ( $\mathrm{B}$, C) Individual changes in serum NfL concentrations from baseline (v1) to follow-up 1 year later (v2) with Wilcoxon test results for patients with behavioral variant frontotemporal dementia (bvFTD) clinically diagnosed with possible bvFTD (B) and definite/probable bvFTD cases (C), respectively. (D, E) Correlation and linear regression for serum NfL and functional scores for bvFTD (red) and Alzheimer disease (AD) (blue) at follow-up after 1 year (V2). In (F), the correlation and linear regression of serum NfL and frontal lobe volumes at follow-up after 1 year (v2) for bvFTD (red) and AD (blue) are shown. (G) Relation between the change in serum NfL and the frontal lobe atrophy from baseline (v1) to follow-up after 1 year (v2) for bvFTD (red) and AD (blue). CDR = Clinical Dementia Rating; $\mathrm{FTLD}=$ frontotemporal lobar degeneration; $\mathrm{MCl}=$ mild cognitive impairment.

In line with the results at baseline examination (visit $1[\mathrm{v} 1]$ ), patients with bvFTD had the highest serum NfL level, for patients with $\mathrm{AD}$ increased levels were determined again, and patients with MCI and controls still had low levels at second follow-up examination (visit 2 [v2]) (figure 2A).

Compared to v1, patients with bvFTD and patients with $\mathrm{AD}$ had increased serum NfL concentrations at v2 (Wilcoxon test: $p=0.0039$ and $p=0.0006$, respectively), whereas levels stayed low for patients with MCI $(p=0.1406)$ and control patients $(p=0.3461)$.

As the individual change of serum NfL from v1 to v2 varied substantially for patients with bvFTD, we examined whether there was a difference in the subgroup of definite/probable vs possible bvFTD cases. We found that in both subgroups, serum NfL levels increased on average with a significant result for the genetic/probable bvFTD subgroup (figure 2, B and $\mathrm{C}$ ).

\section{Correlation of serum NfL with functional scores and brain atrophy at follow-up}

For bvFTD but not $\mathrm{AD}$, the serum NfL level still correlated with the MMSE score (figure 2D), FTLD-CDR-SOB score (figure 2E), and CDR-SOB score ( $r=0.5076$, 95\% CI 0.2960 to $0.6717, p<0.0001$ ), as well as with volumes of several brain regions, including, e.g., the frontal lobe (figure 2F). Overall, the results were similar to those obtained for bvFTD and also for $\mathrm{AD}$ at baseline. A summary of Spearman correlation analysis at $\mathrm{v} 2$ is given in table e-3.

Subgroup analysis of possible and genetic/probable bvFTD for correlation of serum NfL with psychometric and morphometric measurements yielded no difference for the clinical groups and results were similar to those obtained for all bvFTD cases (data not shown).

No correlation was found for the change in NfL levels with the change in functional scores or the change in the degree of 
Table 3 Analysis of behavioral variant frontotemporal dementia (bvFTD) and Alzheimer disease (AD) subgroups selected based on CSF biomarker levels

\begin{tabular}{|c|c|c|c|c|}
\hline & \multicolumn{2}{|c|}{$A \beta 42$ consistent with non- $A D$ vs $A D$} & \multicolumn{2}{|c|}{$A \beta 42$ and tau or $p$-tau consistent with non-AD vs AD } \\
\hline & bvFTD (30) & $A D(11)$ & bvFTD (31) & $A D(14)$ \\
\hline $\begin{array}{l}\text { Serum NfL at v1, mean } \\
\text { (SD) }\end{array}$ & $45.2(25)$ & $25(9.9)$ & $64.6(9.6)$ & $25.3(9.5)$ \\
\hline bvFTD vs AD (v1) & $p=0.0038$ & & \multicolumn{2}{|c|}{$p=0.0015$} \\
\hline Cutoff, pg/mL & 33.0 & & \multicolumn{2}{|l|}{34.3} \\
\hline ROC-AUC & 0.8 & & \multicolumn{2}{|l|}{0.8} \\
\hline Sensitivity \% & 90 & & \multicolumn{2}{|l|}{93} \\
\hline \multirow[t]{2}{*}{ Specificity \% } & \multicolumn{2}{|l|}{67} & \multicolumn{2}{|l|}{61} \\
\hline & $\begin{array}{l}\text { Correlation at } \mathrm{v} 1 \text { of serum NfL } \\
\text { with bvFTD }\end{array}$ & $\begin{array}{l}\text { Correlation at } \mathrm{v} 1 \text { of serum } \\
\mathrm{NfL} \text { with } \mathrm{AD}\end{array}$ & $\begin{array}{l}\text { Correlation at } \mathrm{v} 1 \text { of serum NfL } \\
\text { with bvFTD }\end{array}$ & $\begin{array}{l}\text { Correlation at } \mathrm{v} 1 \text { of serum } \\
\mathrm{NfL} \text { with } \mathrm{AD}\end{array}$ \\
\hline \multirow[t]{2}{*}{ MMSE } & $r=-0.4445$ & $r=-0.1785$ & $r=-0.4425$ & $r=-0.1191$ \\
\hline & $p=0.0156$ & $p=0.5995$ & $p=0.0143$ & $p=0.6852$ \\
\hline \multirow[t]{2}{*}{ CDR-SOB } & $r=0.3404$ & $r=0.4771$ & $r=0.3321$ & $r=0.4747$ \\
\hline & $p=0.0823$ & $p=0.1632$ & $p=0.0843$ & $p=0.1401$ \\
\hline \multirow[t]{2}{*}{ FTLD-CDR-SOB } & $r=0.3539$ & $r=0.4878$ & $r=03527$ & $r=0.4612$ \\
\hline & $p=0.0702$ & $p=0.1526$ & $p=0.0656$ & $p=0.1534$ \\
\hline \multirow[t]{2}{*}{ Cerebrum } & $r=-0.5270$ & $r=0.4333$ & $r=-0.5231$ & $r=0.2727$ \\
\hline & $p=0.0082$ & $p=0.2440$ & $p=0.0073$ & $p=0.4171$ \\
\hline \multirow[t]{2}{*}{ Frontal lobe } & $r=-0.4557$ & $r=0.0167$ & $r=-0.4515$ & $r=-0.0182$ \\
\hline & $p=0.0252$ & $p=0.9661$ & $p=0.0235$ & $p=0.9577$ \\
\hline \multirow[t]{2}{*}{ Temporal lobe } & $r=-0.5226$ & $r=0.1500$ & $r=-0.5169$ & $r=-0.2636$ \\
\hline & $p=0.0088$ & $p=0.7001$ & $p=0.0081$ & $p=0.4334$ \\
\hline \multirow[t]{2}{*}{ Hippocampus } & $r=-0.4209$ & $r=0.2333$ & $r=-0.4262$ & $r=-0.2455$ \\
\hline & $p=0.0406$ & $p=0.5457$ & $p=0.0337$ & $p=0.4669$ \\
\hline \multirow[t]{2}{*}{ CSF NfL } & $r=0.7325$ & $r=0.2636$ & $r=0.7197$ & $r=0.3451$ \\
\hline & $p<0.0001$ & $p=0.4334$ & $p<0.0001$ & $p=0.2269$ \\
\hline \multirow[t]{2}{*}{ CSF pNfH } & $r=0.2884$ & $r=0.00$ & $r=0.2801$ & $r=-0.1123$ \\
\hline & $p=0.1222$ & $p=1.0000$ & $p=0.1270$ & $p=0.7022$ \\
\hline \multirow[t]{2}{*}{ CSF A 442} & $r=-0.2091$ & $r=-0.0909$ & $r=-0.1988$ & $r=-0.0022$ \\
\hline & $p=0.2674$ & $p=0.7904$ & $p=0.2836$ & $p=0.9941$ \\
\hline \multirow[t]{2}{*}{ CSF tau } & $r=0.4874$ & $r=0.5818$ & $r=0.4748$ & $r=0.5209$ \\
\hline & $p=0.0063$ & $p=0.0604$ & $p=0.0070$ & $p=0.0562$ \\
\hline \multirow[t]{2}{*}{ CSF p-tau } & $r=0.1994$ & $r=0.3364$ & $r=0.1906$ & $r=0.3670$ \\
\hline & $p=0.2908$ & $p=0.3118$ & $p=0.3044$ & $p=0.1967$ \\
\hline $\begin{array}{l}\text { Wilcoxon test serum } \\
\text { NfL v1 } \rightarrow \text { V2 }\end{array}$ & $p=0.0002$ & $p=0.0087$ & $p=0.0002$ & $p=0.0021$ \\
\hline $\begin{array}{l}\text { Serum NfL (v2), mean } \\
\text { (SD) }\end{array}$ & $64(42.3)$ & $30.5(13)$ & $63.6(42.6)$ & 31.7 (12.6) \\
\hline bvFTD vs AD (v2) & \multicolumn{2}{|l|}{$p=0.0053$} & \multicolumn{2}{|c|}{$p=0.0041$} \\
\hline Cutoff & \multicolumn{2}{|l|}{41.3} & 45.7 & \\
\hline
\end{tabular}


Table 3 Analysis of behavioral variant frontotemporal dementia (bvFTD) and Alzheimer disease (AD) subgroups selected based on CSF biomarker levels (continued)

\begin{tabular}{|c|c|c|c|c|}
\hline & \multicolumn{2}{|c|}{$A \beta 42$ consistent with non-AD vs $A D$} & \multicolumn{2}{|c|}{$A \beta 42$ and tau or $p$-tau consistent with non-AD vs $A D$} \\
\hline & bvFTD (30) & $A D(11)$ & bvFTD (31) & $A D(14)$ \\
\hline ROC AUC & \multicolumn{2}{|l|}{0.79} & \multicolumn{2}{|l|}{0.77} \\
\hline Sensitivity \% & \multicolumn{2}{|l|}{91} & \multicolumn{2}{|l|}{93} \\
\hline \multirow[t]{2}{*}{ Specificity \% } & \multicolumn{2}{|l|}{68} & \multicolumn{2}{|l|}{61} \\
\hline & $\begin{array}{l}\text { Correlation at } \mathrm{v} 2 \text { of serum NfL } \\
\text { with bvFTD }\end{array}$ & $\begin{array}{l}\text { Correlation at v2 of serum } \\
\text { NfL with } A D\end{array}$ & $\begin{array}{l}\text { Correlation at } \mathrm{v} 2 \text { of serum NfL } \\
\text { with bvFTD }\end{array}$ & $\begin{array}{l}\text { Correlation at } \mathrm{v} 2 \text { of serum } \\
\mathrm{NfL} \text { with } A D\end{array}$ \\
\hline \multirow[t]{2}{*}{ MMSE } & $r=-0.5666$ & $r=-0.4184$ & $r=-0.5735$ & $r=-0.4291$ \\
\hline & $p=0.0017$ & $p=0.2003$ & $p=0.0018$ & $p=0.1105$ \\
\hline \multirow[t]{2}{*}{ CDR-SOB } & $r=0.4191$ & $r=0.0838$ & $r=0.4214$ & $r=0.5654$ \\
\hline & $p=0.0236$ & $p=0.8435$ & $p=0.0286$ & $p=0.0554$ \\
\hline \multirow[t]{2}{*}{ FTLD-CDR-SOB } & $r=0.4177$ & $r=0.1905$ & $r=0.4192$ & $r=0.6175$ \\
\hline & $p=0.0270$ & $p=0.6514$ & $p=0.0330$ & $p=0.0324$ \\
\hline \multirow[t]{2}{*}{ Cerebrum } & $r=-0.4353$ & $r=0.3714$ & $r=-0.4750$ & $r=0.2143$ \\
\hline & $p=0.0920$ & $p=0.4685$ & $p=0.0736$ & $p=0.6103$ \\
\hline \multirow[t]{2}{*}{ Frontal lobe } & $r=-0.3824$ & $r=0.7714$ & $r=-0.3964$ & $r=0.3333$ \\
\hline & $p=0.1439$ & $p=0.0724$ & $p=0.1435$ & $p=0.4198$ \\
\hline \multirow[t]{2}{*}{ Temporal lobe } & $r=-0.6706$ & $r=-0.1429$ & $r=-0.6857$ & $r=0.1429$ \\
\hline & $p=0.0045$ & $p=0.7872$ & $p=0.0048$ & $p=0.7358$ \\
\hline \multirow[t]{2}{*}{ Hippocampus } & $r=-0.3941$ & $r=-0.1429$ & $r=-0.3857$ & $r=-0.2143$ \\
\hline & $p=0.1309$ & $p=0.7872$ & $p=0.1556$ & $p=0.6103$ \\
\hline
\end{tabular}

Abbreviations: $A \beta 42=\beta$-amyloid 1-42; $A \cup C=$ area under the curve; CDR-SOB = Clinical Dementia Rating scale Sum of Boxes; FTLD = frontotemporal lobar degeneration; MMSE = Mini-Mental State Examination; NfL = neurofilament light chain; $\mathrm{p}$-tau = phosphorylated tau; pNfH = phosphorylated neurofilament heavy chain; ROC = receiver operating characteristic; v1 = visit 1; v2 = visit 2.

regional brain atrophy, when looking at absolute or normalized concentration/volume changes (as example, the frontal lobe is shown in figure $2 \mathrm{G}$ ).

\section{Serum NfL in patients with genetic bvFTD with GRN, MAPT, or C9orf72 mutation/expansion}

Looking at the demographic characteristics, functional scores, regional brain volumes, and CSF marker levels, no remarkable differences could be observed between the identified genetic cases and patients tested negative or patients not tested. The patient with novel splice site mutation in GRN had a relatively high serum NfL level that was further increased at follow-up (v1: $134 \mathrm{pg} / \mathrm{mL}, \mathrm{v} 2: 149 \mathrm{pg} / \mathrm{mL}$ ) and also the patient with MAPT mutation, who had an intermediate NfL level $(55 \mathrm{pg} /$ $\mathrm{mL}$ ), showed increasing concentrations over time (v2: $77 \mathrm{pg}$ ) $\mathrm{mL}$, visit 3: $98 \mathrm{pg} / \mathrm{mL}$ ). For the 7 patients with $C 9$ orf 72 repeat expansion, mean $\mathrm{NfL}$ serum level was in the range of all bvFTD (v1: $45.6 \mathrm{pg} / \mathrm{mL}$ ) and increased on average (v2: 50.4 $\mathrm{pg} / \mathrm{mL})$.

\section{Serum NfL in patients with bvFTD and patients with AD selected on the basis of CSF AD core biomarkers}

Because of discrepancies between clinical diagnosis of bvFTD and $\mathrm{AD}$ on the one hand, and levels of the CSF $\mathrm{AD}$ core biomarkers on the other hand, we performed additional analyses with a group definition based on CSF biomarkers (table 3). By excluding patients with bvFTD with decreased $A \beta 42$ and patients with $A D$ with normal $A \beta 42$, the difference between serum $\mathrm{NfL}$ levels in the 2 diagnosis groups slightly increased and ROC analysis yielded an area under the curve (AUC) of 0.8. At a serum NfL cutoff of $33 \mathrm{pg} / \mathrm{mL}$, the sensitivity improved to $90 \%$ and the specificity to $67 \%$. While in the bvFTD group at baseline, serum NfL still correlated with the MMSE $(r=-0.4445, p=0.0156)$, the association with the CDR and the FTLD-CDR barely failed to be significant. The correlation of serum NfL with temporal lobe volumes was more pronounced $(r=-0.5226, p=0.0088)$ and with frontal lobe volumes less marked $(r=-0.4557, p=0.0252)$. For the 
$\mathrm{AD}$ group, we still did not observe a correlation with functional scores or regional brain volumes. Analysis of follow-up data yielded similar results, i.e., a better discriminatory power of serum NfL for discrimination of bvFTD and $\mathrm{AD}$, and correlation of serum $\mathrm{Nfl}$ with global functional scores and regional brain volumes for bvFTD but not $\mathrm{AD}$.

We then defined subgroups on the basis of both CSF A $\beta 42$ and tau levels and excluded patients with bvFTD with combined decreased $\mathrm{A} \beta 42$ and increased tau or p-tau. Accordingly, patients with $\mathrm{AD}$ without the typical $\mathrm{AD}$ CSF biomarker pattern were excluded from the analyses as well. By ROC analysis, we calculated an AUC of 0.80 and a sensitivity of $93 \%$ and specificity of $61 \%$ at a serum NfL cutoff value of $34.3 \mathrm{pg} /$ $\mathrm{mL}$. Coefficients for the correlation of serum NfL with clinical scores and atrophy were very similar to the values calculated for the cohort defined on the basis of CSF A $\beta 42$ only. For both subcohorts, we still determined increased serum NfL at follow-up and the increase was more pronounced for bvFTD and less marked for $\mathrm{AD}$ compared to the entire cohort.

\section{Discussion}

Analyzing 74 patients with clinically diagnosed bvFTD at baseline and at follow-up, we could show that serum NfL level is increased if compared with controls, patients with MCI, and patients with $\mathrm{AD}$, increased on average over time, and correlated at both examination dates with functional scores and brain volumes of mainly frontal cortical as well as subcortical regions well known to be specifically impaired in bvFTD. ${ }^{4,5}$ In contrast, the analysis of 26 patients with $\mathrm{AD}$, who had higher serum NfL levels compared to patients with MCI or controls, revealed no association of serum NfL and functional or volumetric measures of disease severity.

An increase of NfL in CSF and association of CSF NfL levels with disease severity or progression was observed before in neurodegenerative diseases such as ALS, ${ }^{12,24,30,31} \mathrm{AD},{ }^{11,32,33}$ CreutzfeldtJakob disease, ${ }^{34,35}$ and FTLD. ${ }^{21,33,36-38}$ Studies investigating serum NfL showed that levels were strongly associated with NfL levels in the CSF. ${ }^{16,17,20,39}$ In the present study, we could show this for the first time for a large cohort of patients with bvFTD.

We found that serum NfL correlates with the MMSE score quantifying cognitive impairment, which is in line with a recent article analyzing serum samples of a cohort comprising 74 patients with FTLD, half of whom were diagnosed with bvFTD ${ }^{21}$ Furthermore, in support of the clinical relevance of $\mathrm{NfL}$ for bvFTD, we also found a correlation with the CDR$\mathrm{SOB}$, in line with data obtained measuring FTD CSF samples, ${ }^{36}$ and with the FTLD-CDR-SOB, a functional score established to specifically assess disease severity in FTLD subgroups. The fact that the correlation between serum NfL and the functional performance persisted at follow-up examination points to the usefulness of serum NfL as monitoring marker at different disease stages.
In our bvFTD cohort, serum NfL correlated with brain volumetric measures, which is similar to what could be described for CSF NfL ${ }^{36}$ and could have been expected because of the correlation of NfL levels in CSF and blood. A different study ${ }^{21}$ of patients with FTD did not find an association with volumes but with the frontal lobe atrophy rate, which was interpreted as serum NfL being a marker of severity rather than disease duration. For our cohort comprising solely patients with bvFTD, we could not find an association with disease duration and no correlation with the progression of atrophy. As the disease duration at baseline in our study relies on the date of symptom onset reported by the patient or caregiver and considering the slowly progressive nature of bvFTD, however, the significance of our results might be limited.

For primary progressive aphasia, where we found a larger increase of serum NfL during progression than for bvFTD in the present study, we could report on the correlation of serum NfL concentration change with the atrophy progression rate and not with brain volumes at the time of serum sampling. ${ }^{20}$ In addition, for progressive supranuclear palsy and corticobasal syndrome, no correlation of white matter lesions with serum NfL was found. ${ }^{16}$ Thus there is evidence for differences present in serum NfL level dynamics and time courses during progression of the different clinical FTLD subgroups. Whether this is due to the underlying pathology needs further clinical-toneuropathologic studies. As for the vast majority of patients included in our ongoing multicenter FTLD consortium, the molecular pathology is not yet assessed; it is a limitation of our study that analysis of the relation of serum NfL to the underlying molecular pathology could not be carried out. A recent study described similar CSF NfL levels in definite FTLD with different molecular pathology, ${ }^{40}$ which might argue against a different influence of TDP-43 and tau on NfL level. However, as in our study, the diagnostic power of the discrimination between bvFTD and $\mathrm{AD}$ improved by excluding patients with bvFTD with AD-typical CSF A 442 concentrations; it might be that serum NfL is influenced differently by FTLD (TDP-43, tau) or $\mathrm{AD}(\mathrm{A} \beta 42)$ pathology. Also the fact that the analysis of our $\mathrm{AD}$ group, in contrast to results obtained by others, ${ }^{17} \mathrm{did}$ not reveal any association of NfL with brain atrophy, although we observed the reported pattern of degeneration compared to bvFTD, ${ }^{41,42}$ could speak for NfL as a pathology-dependent marker. An alternative interpretation would be that atrophy of specific brain regions is more reflected in raised serum NfL levels than atrophy of others. Our data indicate that atrophy in frontal cortical brain regions, the striatum, and other subcortical regions contribute more to the CSF and serum NfL level than degeneration of parietal regions. To clarify these questions, studies comprising more patients with different patterns of atrophy and neuropathologically characterized cases have to be analyzed in comparison. Our study involved a smaller number of patients with $\mathrm{AD}$ than patients with bvFTD.

About $15 \%$ of patients with FTD develop ALS in the course of the disease ${ }^{43}$ and patients with ALS as well as FTD-ALS are known to have markedly higher serum NfL levels than 
patients with FTD. ${ }^{21,22}$ The increase seems to happen just before or at ALS symptom onset ${ }^{44}$; hence we suppose that serum NfL could be useful not only for monitoring bvFTD severity but also has the potential to detect ALS in bvFTD. The patients in this study did not develop motor symptoms over the time course observed, but it would be interesting to monitor motor symptoms in further follow-up.

We provide evidence for NfL being a blood biomarker reflecting functional impairment as well as degree of atrophy of patients with bvFTD at different stages of disease. NfL might therefore be helpful to monitor effects in upcoming therapeutic trials targeting neurodegeneration and functional performance of patients with bvFTD.

\section{Author contributions}

All authors made substantial contributions to conception and design, acquisition of data, or analysis and interpretation of data. All authors gave final approval of the version to be submitted and agree to be accountable for all aspects of the work in ensuring that questions related to the accuracy or integrity of any part of the work are appropriately investigated and resolved. Conception and design of the study: P. Steinacker, M. Otto; sample collection, phenotyping, and data management: P. Steinacker, S. Anderl-Straub, J. DiehlSchmid, E.S. Dipl-Psych, I. Uttner, C.A.F. von Arnim, H. Barthel, A. Danek, K. Fassbender, K. Fliessbach, H. Foerstl, T. Grimmer, H-JH, H Jahn, J. Kassubek, J. Kornhuber, B. Landwehrmeyer, M. Lauer, J.M. Maler, B. Mayer, P. Oeckl, J. Prudlo, A. Schneider, A.E. Volk, J. Wiltfang, M.L. Schroeter, A.C. Ludolph, M. Otto; study management and coordination: P. Steinacker, M. Otto, S. Anderl-Straub, E.S. Dipl-Psych, J. Diehl-Schmid, A. Danek, A.C. Ludolph; statistical methods and analysis: P. Steinacker, H.J. Huppertz, B. Mayer; interpretation of results: P. Steinacker, M. Otto; manuscript writing: P. Steinacker, M. Otto; critical revision of the manuscript: P. Steinacker, S. Anderl-Straub, J. Diehl-Schmid, E.S. Dipl-Psych, I. Uttner, C.A.F. von Arnim, H. Barthel, A. Danek, K. Fassbender, K. Fliessbach, H. Foerstl, T. Grimmer, H.J. Huppertz, H Jahn, J. Kassubek, J. Kornhuber, B. Landwehrmeyer, M. Lauer, J.M. Maler, B. Mayer, P. Oeckl, J. Prudlo, A. Schneider, A.E. Volk, J. Wiltfang, M.L. Schroeter, A.C. Ludolph, M. Otto.

\section{Acknowledgment}

The authors thank the patients for participating in this study.

\section{Study funding}

The study was supported by grants from the German Federal Ministry of Education and Research (project: FTLDc 01GI1007A), the EU Joint Programme-Neurodegenerative Disease Research (JPND) network, PreFrontAls (01ED1512), the Foundation of the State of Baden-Württemberg (D.3830), the Thierry Latran Foundation, and BIU (D.5009). Prof. Jens Wiltfang is supported by an Ilídio Pinho professorship and iBiMED (UID/BIM/04501/2013), at the University of Aveiro, Portugal.

\section{Disclosure}

P. Steinacker, S. Anderl-Straub, J. Diehl-Schmid, E. Semler, I. Uttner, and C. von Arnim report no disclosures with regard to the submitted work. Outside the submitted work, Prof. von Arnim received honoraria from serving on the scientific advisory board of Nutricia $\mathrm{GmbH}$ and Honkong University Research council and has received funding for travel and speaker honoraria from Nutricia GmbH, Lilly Deutschland $\mathrm{GmbH}$, Desitin Arzneimittel GmbH, Biogen, Roche, and Dr. Willmar Schwabe GmbH \& Co. KG. H. Barthel, A. Danek, K. Fassbender, K. Fliessbach, H. Foerstl, and T. Grimmer report no disclosures with regards to the submitted work. Outside the submitted work, Dr. Grimmer reported having received consulting fees from Actelion, Eli Lilly, Iqvia/ Quintiles, MSD, Novartis, Quintiles, and Roche Pharma; lecture fees from Biogen, Lilly, Parexel, and Roche Pharma; and grants to his institution from Actelion and PreDemTech. H. Huppertz, H. Jahn, J. Kassubek, J. Kornhuber, G. Landwehrmeyer, M. Lauer, J. Maler, B. Mayer, P. Oeckl, J. Prudlo, A. Schneider, A. Volk, and J. Wiltfang report no disclosures with regards to the submitted work. Outside the submitted work, Prof. Wiltfang reported having received consulting fees from Boehringer-Ingelheim, Eli Lilly, and MSD Sharp \& Dohme; and lecture fees from Hoffmann-LaRoche. M. Schroeter, A. Ludolph, and M. Otto report no disclosures relevant to the manuscript. Go to Neurology.org/ $\mathrm{N}$ for full disclosures.

\section{Publication history}

Received by Neurology January 12, 2018. Accepted in final form July 10, 2018.

\section{References}

1. Rosso SM, Donker Kaat L, Baks T, et al. Frontotemporal dementia in the Netherlands: patient characteristics and prevalence estimates from a population-based study. Brain 2003;126:2016-2022.

2. Rabinovici GD, Miller BL. Frontotemporal lobar degeneration: epidemiology, pathophysiology, diagnosis and management. CNS Drugs 2010;24:375-398.

3. Rascovsky K, Hodges JR, Knopman D, et al. Sensitivity of revised diagnostic criteria for the behavioural variant of frontotemporal dementia. Brain 2011;134: 2456-2477.

4. Schroeter ML, Laird AR, Chwiesko C, et al. Conceptualizing neuropsychiatric diseases with multimodal data-driven meta-analyses: the case of behavioral variant frontotemporal dementia. Cortex 2014;57:22-37.

5. Schroeter ML, Raczka K, Neumann J, Yves von Cramon D. Towards a nosology for frontotemporal lobar degenerations: a meta-analysis involving 267 subjects. Neuroimage 2007;36:497-510.

6. Cairns NJ, Bigio EH, Mackenzie IR, et al. Neuropathologic diagnostic and nosologic criteria for frontotemporal lobar degeneration: consensus of the Consortium for Frontotemporal Lobar Degeneration. Acta Neuropathol 2007; 114:5-22.

7. Josephs KA, Hodges JR, Snowden JS, et al. Neuropathological background of phenotypical variability in frontotemporal dementia. Acta Neuropathol 2011;122: 137-153.

8. Tsai RM, Boxer AL. Therapy and clinical trials in frontotemporal dementia: past, present, and future. J Neurochem 2016;138(suppl 1):211-221.

9. Pijnenburg YA, Verwey NA, van der Flier WM, Scheltens P, Teunissen CE. Discriminative and prognostic potential of cerebrospinal fluid phospho-tau/tau ratio and neurofilaments for frontotemporal dementia subtypes. Alzheimers Dement (Amst) 2015;1:505-512.

10. Bagnato S, Grimaldi LME, Di Raimondo G, et al. Prolonged cerebrospinal fluid neurofilament light chain increase in patients with post-traumatic disorders of consciousness. J Neurotrauma 2017;34:2475-2479.

11. Mattsson N, Insel PS, Palmqvist $S$, et al. Cerebrospinal fluid tau, neurogranin, and neurofilament light in Alzheimer's disease. EMBO Mol Med 2016;8:1184-1196.

12. Steinacker P, Feneberg E, Weishaupt J, et al. Neurofilaments in the diagnosis of motoneuron diseases: a prospective study on 455 patients. J Neurol Neurosurg Psychiatry 2016;87:12-20. 
13. Malmestrom C, Haghighi S, Rosengren L, Andersen O, Lycke J. Neurofilament light protein and glial fibrillary acidic protein as biological markers in MS. Neurology 2003; 61:1720-1725.

14. Gaiottino J, Norgren N, Dobson R, et al. Increased neurofilament light chain blood levels in neurodegenerative neurological diseases. PLoS One 2013;8:e75091.

15. Rissin DM, Kan CW, Campbell TG, et al. Single-molecule enzyme-linked immunosorbent assay detects serum proteins at subfemtomolar concentrations. Nat Biotechnol 2010;28:595-599.

16. Hansson O, Janelidze S, Hall S, et al. Blood-based NfL: a biomarker for differential diagnosis of parkinsonian disorder. Neurology 2017;88:930-937.

17. Mattsson N, Andreasson U, Zetterberg H, Blennow K. Association of plasma neurofilament light with neurodegeneration in patients with Alzheimer disease. JAMA Neurol 2017;74:557-566.

18. Rojas JC, Karydas A, Bang J, et al. Plasma neurofilament light chain predicts progression in progressive supranuclear palsy. Ann Clin Transl Neurol 2016;3:216-225.

19. Steinacker P, Huss A, Mayer B, et al. Diagnostic and prognostic significance of neurofilament light chain NF-L, but not progranulin and S100B, in the course of amyotrophic lateral sclerosis: data from the German MND-net. Amyotroph Lateral Scler Frontotemporal Degener 2017;18:112-119.

20. Steinacker P, Semler E, Anderl-Straub S, et al. Neurofilament as a blood marker for diagnosis and monitoring of primary progressive aphasias. Neurology 2017;88: 961-969.

21. Rohrer JD, Woollacott IO, Dick KM, et al. Serum neurofilament light chain protein is a measure of disease intensity in frontotemporal dementia. Neurology 2016;87: 1329-1336.

22. Wilke C, Preische O, Deuschle C, et al. Neurofilament light chain in FTD is elevated not only in cerebrospinal fluid, but also in serum. J Neurol Neurosurg Psychiatry 2016;87:1270-1272.

23. Meeter LH, Dopper EG, Jiskoot LC, et al. Neurofilament light chain: a biomarker for genetic frontotemporal dementia. Ann Clin Transl Neurol 2016;3:623-636.

24. Lu CH, Macdonald-Wallis C, Gray E, et al. Neurofilament light chain: a prognostic biomarker in amyotrophic lateral sclerosis. Neurology 2015;84:2247-2257.

25. Otto M, Ludolph AC, Landwehrmeyer B, et al. German consortium for frontotemporal lobar degeneration. Nervenarzt 2011;82:1002-1005.

26. McKhann GM, Knopman DS, Chertkow H, et al. The diagnosis of dementia due to Alzheimer's disease: recommendations from the National Institute on Aging-Alzheimer's Association workgroups on diagnostic guidelines for Alzheimer's disease. Alzheimers Dement 2011;7:263-269.

27. Albert MS, DeKosky ST, Dickson D, et al. The diagnosis of mild cognitive impairment due to Alzheimer's disease: recommendations from the National Institute on Aging-Alzheimer's Association workgroups on diagnostic guidelines for Alzheimer's disease. Alzheimers Dement 2011;7:270-279.

28. Huppertz HJ, Kroll-Seger J, Kloppel S, Ganz RE, Kassubek J. Intra- and interscanner variability of automated voxel-based volumetry based on a 3D probabilistic atlas of human cerebral structures. Neuroimage 2010;49:2216-2224.
29. Shattuck DW, Mirza M, Adisetiyo V, et al. Construction of a 3D probabilistic atlas of human cortical structures. Neuroimage 2008;39:1064-1080.

30. Oeckl PP, Jardel CPP, Salachas FM, et al. Multicenter validation of CSF neurofilaments as diagnostic biomarkers for ALS. Amyotroph Lateral Scler Frontotemporal Degener 2016;17:404-413.

31. Rosengren LE, Karlsson JE, Karlsson JO, Persson LI, Wikkelso C. Patients with amyotrophic lateral sclerosis and other neurodegenerative diseases have increased levels of neurofilament protein in CSF. J Neurochem 1996;67:2013-2018.

32. Zetterberg H, Skillback T, Mattsson N, et al. Association of cerebrospinal fluid neurofilament light concentration with Alzheimer disease progression. JAMA Neurol 2016;73:60-67.

33. Sjogren M, Rosengren L, Minthon L, Davidsson P, Blennow K, Wallin A. Cytoskeleton proteins in CSF distinguish frontotemporal dementia from AD. Neurology 2000; 54:1960-1964.

34. van Eijk JJ, van Everbroeck B, Abdo WF, Kremer BP, Verbeek MM. CSF neurofilament proteins levels are elevated in sporadic Creutzfeldt-Jakob disease. J Alzheimers Dis 2010;21:569-576.

35. Steinacker P, Blennow K, Halbgebauer S, et al. Neurofilaments in blood and CSF for diagnosis and prediction of onset in Creutzfeldt-Jakob disease. Sci Rep 2016;6:38737.

36. Scherling CS, Hall T, Berisha F, et al. Cerebrospinal fluid neurofilament concentration reflects disease severity in frontotemporal degeneration. Ann Neurol 2014;75:116-126.

37. Skillback T, Mattsson N, Blennow K, Zetterberg H. Cerebrospinal fluid neurofilament light concentration in motor neuron disease and frontotemporal dementia predicts survival. Amyotroph Lateral Scler Frontotemporal Degener 2017;18:1-7.

38. Landqvist Waldo M, Frizell Santillo A, Passant U, et al. Cerebrospinal fluid neurofilament light chain protein levels in subtypes of frontotemporal dementia. BMC Neurol 2013;13:54.

39. Kuhle J, Barro C, Andreasson U, et al. Comparison of three analytical platforms for quantification of the neurofilament light chain in blood samples: ELISA, electrochemiluminescence immunoassay and Simoa. Clin Chem Lab Med 2016;54: $1655-1661$.

40. Goossens J, Bjerke M, Van Mossevelde S, et al. Diagnostic value of cerebrospinal fluid tau, neurofilament, and progranulin in definite frontotemporal lobar degeneration. Alzheimers Res Ther 2018;10:31.

41. Landin-Romero R, Kumfor F, Leyton CE, Irish M, Hodges JR, Piguet O. Diseasespecific patterns of cortical and subcortical degeneration in a longitudinal study of Alzheimer's disease and behavioural-variant frontotemporal dementia. Neuroimage 2017;151:72-80.

42. Schroeter ML, Stein T, Maslowski N, Neumann J. Neural correlates of Alzheimer's disease and mild cognitive impairment: a systematic and quantitative meta-analysis involving 1351 patients. Neuroimage 2009;47:1196-1206.

43. Lomen-Hoerth C, Anderson T, Miller B. The overlap of amyotrophic lateral sclerosis and frontotemporal dementia. Neurology 2002;59:1077-1079.

44. Feneberg E, Oeckl P, Steinacker P, et al. Multicenter evaluation of neurofilaments in early symptom onset amyotrophic lateral sclerosis. Neurology 2018;90:e22-e30. 